\title{
Soziale Medien in der Chirurgie?
}

\section{Social Media in Surgery?}

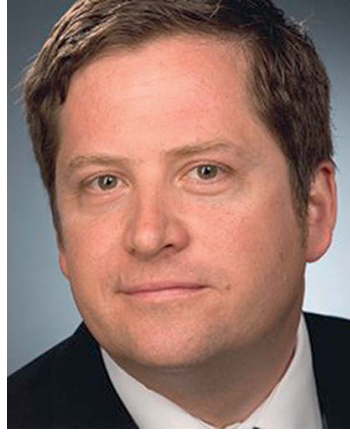

Univ.-Prof. Dr. med. Riccardo Giunta
Bibliografie

DOI http://dx.doi.org/

10.1055/s-0035-1559681

Handchir Mikrochir Plast Chir

2015; 47: 211-212

(c) Georg Thieme Verlag KG

Stuttgart · New York

ISSN 0722-1819

Korrespondenzadresse Univ.-Prof. Dr. med. Riccardo Giunta

Handchirurgie, Plastische Chirurgie und Ästhetische Chirurgie Campus Innenstadt und Großhadern der Ludwig-Maximilians Universität München

Pettenkoferstraße 8a 80336 München

r.giunta@med.uni-muenchen.de
Liebe Leserinnen und Leser,

Soziale Medien sind von unserer Informationsgesellschaft als Massenmedium heutzutage nicht mehr wegzudenken. Mit relativ einfachen Mitteln wie einem „Smartphone“ oder einem „Tablet Computer" kann man sich zu jeder Zeit an einem globalen Netzwerk beteiligen und multimediale Informationen empfangen und auch sofort veröffentlichen. Im Gegensatz zu herkömmlichen Massenmedien wie Zeitung, Radio oder Fernsehen ist der Informationsfluss nicht einseitig und auch nicht an eine lineare Zeitschiene gebunden. Im Grunde kann jeder zu jeder Zeit Informationen verbreiten und auch nutzen, sodass die meisten von uns sich wegen der Aktualität und Vernetzung in unserem privaten Alltag schon an die Nutzung gewöhnt haben. Wer nutzt Soziale Medien und wo und wie werden sie in der Medizin und in der Plastischen Chirurgie und Handchirurgie angewendet?

Unter den niedergelassenen Plastischen Chirurgen in den USA nutzten in einer Online Umfrage etwa die Hälfte Soziale Medien [1]. Erwartungsgemäß hat mehr als die Hälfte von diesen das Medium als Marketing Instrument, also für den Arzt-Patienten Kontakt, eingesetzt. Am häufigsten wurden demnach in den USA Facebook ${ }^{\circledR}$, LinkedIn $^{\circledR}$, Twitter $^{\circledR}$ und YouTube ${ }^{\circledR}$ benutzt. Diejenigen, die in dieser Studie Soziale Medien nicht nutzten, waren am häufigsten darüber besorgt, mit der Nutzung die berufliche Distanz zum Patienten zu stark zu reduzieren, die Ärztliche Schweigepflicht zu verletzen oder wurden vom zeitlichen Aufwand abgeschreckt.

Natürlich bieten die neue Medien, nicht nur Chancen sondern auch Risiken: Grundsätzlich gilt es unter anderem auch den Datenschutz zu beachten, keine berufswidrige Werbung zu verbreiten und das Fernbehandlungsverbot einzuhalten [2].

Begonnen hat der Trend etwa 2012/2013. Mittlerweile nutzen etwa $65 \%$ der Kliniken in einer Umfrage an 53 Krankenhäusern in Deutschland ebenfalls Soziale Medien als Kommunikationskanal [3]. Am häufigsten wurden in dieser Studie Facebook $^{\circledR}$ und YouTube $^{\circledR}$ genutzt, gefolgt von Xing $^{\circledR}$. Demnach wird Kliniken empfohlen, Soziale Medien in ein Gesamtkommunikationskonzept einzubauen, um sie als Marketing Chance zu verwenden.

Aus unseren eigenen Erfahrung mit unserer Einheit in München ist weniger die Arzt-Patienten Kommunikation der eigentlich Vorteil von Soziale Medien, sondern vielmehr die Arzt-zu-Arzt-
Kommunikation in einer „Peer Community“ und der damit verbundenen engen Einbeziehung von Fachkollegen im In-und Ausland sowie besonders interessierten Medizinstudenten. Die beiden wesentlichen Vorteile Sozialer Medien sind aus unserer Sicht dabei die Aktualität der Information, die von anderen Medien unerreicht ist, und gleichzeitig die konkrete wechselseitige Auswahl der Zielgruppe, sodass die Information auch wesentlich besser Ihren Adressaten findet. Dadurch lassen sich bspw. aktuelle Lehrveranstaltungen oder Symposien sofort an das interessierte Publikum kommunizieren. Genauso können etwa aktuelle Informationen aus dem Fachgebiet, die der Nutzer Sozialer Medien selbst im Netz erhalten hat und für mitteilenswert erachtet werden, per Mausklick sofort an andere Interessierte weiter gegeben werden. Letztlich wird dadurch eine Art Informationsvorsprung bewirkt. Ob dieser am Ende allerdings tatsächlich einen Vorteil bringt, ist natürlich von der Qualität der Information abhängig.

Auch in Lehre und Forschung können Soziale Medien neue Möglichkeiten bieten. Viele Zeitschriften wie etwa die Zeitschrift Plastic and Reconstructive Surgery veröffentlichen in regelmäßigen Abständen Hinweise auf aktuelle Publikationen und Heftinhalte. Darüber hinaus gibt es auch eine ganze Reihe von Videos auf YouTube ${ }^{\circledR}$, die z. B. die spezielle angewandte Anatomie unseres Fachgebiets oder auch Operationstechniken sehr instruktiv erläutern.

Ein rein wissenschaftliches Netzwerk stellt Rese$\operatorname{archGate}^{\circledR}$ dar. Wissenschaftler können hier Ihre Publikationen über Datenbanken suchen, archivieren und so eine Online-Publikationsliste erstellen. Arbeiten können auch hochgeladen werden, allerdings muss beachtet werden, dass das Urheberrecht bzw. das Nutzungsrecht der Verlage dies erlaubt. Die Plattform bewertet den Wissenschaftler mit dem sogenannten „RG-Score“, dessen genaue Berechnungsformel unbekannt ist und von daher vielfältig kritisiert wird. Hauptkritikpunkt ist, dass die Plattform Koautoren eines registrierten Autors in seinem Namen per Email einlädt sich zu registrieren. Damit wird der Eindruck erweckt, der Autor hätte dies veranlasst, obwohl sie ohne sein Zutun verschickt werden. Auch Fachgesellschaften verwenden Soziale Medien immer mehr um ihre Mitglieder zu erreichen. Gleichzeitig können sie auch im Sinne einer modernen Öffentlichkeitsarbeit bspw. Inhalte des Fachgebiets und aktuelle Informationen aus dem Fachgebiet direkt an den Patienten kommunizieren. Die Fachgesellschaften haben hier nicht 
nur die Möglichkeit, sondern auch die Aufgabe das Fachgebiet im Sinne der wichtigen Patienteninformation und -aufklärung kompetent zu vertreten. Gerade Patienten wenden digitale Medien immer mehr an, um sich über ihre Erkrankung und Behandlungsmöglichkeiten zu informieren und sich einen kompetenten Arzt zu suchen. Neben der Webseite gewinnen Soziale Medien immer mehr darin an Bedeutung sich einen ersten Eindruck über den ausgewählten Arzt zu verschaffen, ohne ihn persönlich aufsuchen zu müssen.

Die Anwendungsmöglichkeiten Sozialer Medien sind vielfältig, längst noch nicht ausgeschöpft und die Kreativität hoch. Durch den Wandel unserer Kommunikations- und Informationswege ist zu erwarten, dass immer mehr von uns nicht nur im privaten Bereich, sondern auch in allen Bereichen der Plastischen Chirurgie und Handchirurgie zunehmend auch Soziale Medien nutzen werden, wobei vor allem die Chancen vielfältig, auf den ersten Blick vielleicht gar nicht ersichtlich sind und einem erst bei der tatsächlichen Nutzung bewusst werden. Natürlich bergen Neuerungen immer auch Risiken, aber wie bei jeder Innovation finden wir die Grenzen des eigentlichen Nutzen nur wenn wir sie einsetzen.

München im August 2015

Riccardo Giunta

\section{Literatur}

1 Vardanian AJ, Kusnezov N, Im DD et al. Social Media Use and Impact on Plastic Surgery Practice. Plast Reconstr Surg 2013; 131: 1184

2 Hartz T, Fangerau H, Albrecht UV. Social Media in der ärztlichen Praxis - Chancen, Risiken, Trends. Dtsch Ärztebl 2014; 111: 6-10

3 Lüthy A, Jendreck K. Kommunikation: Social Media - auch hierzulande für Krankenhäuser attraktiv? Dtsch Arztebl 2015; 112: A-276-A-278 